\title{
STARCH SULFURIC ACID: AN ALTERNATIVE, ECO-FRIENDLY CATALYST FOR BIGINELLI REACTION
}

\author{
Ramin Rezaei ${ }^{\mathrm{a}}$, Sara Malek ${ }^{\mathrm{a}}$, Mohammad Reza Sheikhi ${ }^{\mathrm{a}}$, Mohammad Kazem Mohammadi ${ }^{\mathrm{b}}$ \\ ${ }^{a}$ Department of Chemistry, Firoozabad Branch, Islamic Azad University, P.O. Box 74715-117 Firoozabad, Iran \\ ${ }^{b}$ Department of Chemistry, Ahvaz Branch, Islamic Azad University, Ahvaz, Iran \\ *e-mail:rezaieramin@yahoo.com
}

\begin{abstract}
The one-pot multicomponent synthesis of 3,4-dihydropyrimidinone derivatives using starch sulfuric acid as an environmentally friendly biopolymer-based solid acid catalyst from aldehydes, $\beta$-keto esters and urea/ thiourea without solvent is described. Compared with classical Biginelli reaction conditions, this new method has the advantage of minimizing the cost operational hazards and environmental pollution, good yields, shorter reaction times and simple work-up.
\end{abstract}

Keywords: Biginelli reaction, dihydropyrimidinones, starch sulfuric acid, solvent-free, biodegradable catalyst.

\section{Introduction}

Dihydropyrimidinones are attractive organic compounds which show important biological activities such as antiviral, antitumor, antibacterial and anti-inflammatory actions [1-3]. The more convenient procedure for the preparation of dihydropyrimidinones first reported by P. Biginelli in 1893, consists of the one-pot condensation of $\beta$-dicarbonyl compounds with aldehydes and urea or thiourea under strongly acidic conditions [4]. One major disadvantage of this method is the low yields especially in the case of aliphatic and some substituted aromatic aldehydes. To circumvent this problem, a variety of new catalysts has been introduced in the literature. In recent years, many protocols involving the use of Lewis and Bronsted acids such as graphite supported lanthanum chloride, [5] alumina supported $\mathrm{MoO}_{3}$ [6], antimony(III) chloride [7], copper(II) tetrafluoroborate [8], bismuth nitrate [9], iron(III) trifluoroacetate and trifluoromethanesulfonate [10], yttrium(III) nitrate hexahydrate [11], $\mathrm{TaBr}_{5}$ [12], Trichloroisocynuric acid (TCCA) [13], PSSA [14], chloroacetic acid [15], $p$-TsOH [16], $\mathrm{HCl}$ [17], acetic acid [18], silica sulfuric acid [19], concentrated $\mathrm{H}_{2} \mathrm{SO}_{4}$ [20], $\mathrm{H}_{3} \mathrm{BO}_{3}$ [21], $\mathrm{HBF}_{4}$ [22], chiral phosphoric acid [23], $\mathrm{H}_{3} \mathrm{PW}_{12} \mathrm{O}_{40}$ [24], $\mathrm{H}_{3} \mathrm{PMo}_{12} \mathrm{O}_{40}$ [25], $\mathrm{Al}_{2} \mathrm{O}_{3} \mathrm{MeSO}_{3} \mathrm{H}$ [26], $\mathrm{HClO}_{4}$ doped silica [27], bentonite/ $\mathrm{PS}_{-} \mathrm{SO}_{3} \mathrm{H}$ nanocomposite [28], sulfated tungstate [29], imidazol-1-yl-acetic acid [30], zeolite-supported HPA [31] and a number of other catalysts [32-38] are reported. However, at a practical level, these often require relatively harsh reaction conditions such as high reaction temperatures, expensive or highly acidic catalysts, and prolonged reaction times. In most of the cases stoichiometric amounts of catalyst are required in order to achieve good yields. In addition, most of the reactions require tedious work-up procedures and column purification, which ultimately results in diminished yields. Therefore, in spite of a large number of methods reported for this transformation, there is still need to develop a more efficient, simple, milder protocol using conservational catalyst.

In recent years, the direction of science and technology has been shifting more towards eco-friendly, natural product resources and reusable catalysts. Thus, natural biopolymers are attractive candidates in the search for such solid support catalysts [39]. Starch as a one of the most common and easy to recover biopolymer become in the scope of many research $[40,41]$.

We now report an efficient catalyzed method for the synthesis of dihydropyrimidinones via the three-component reaction of $\beta$-dicarbonyl compounds with aldehydes and urea or thiourea under mild conditions (Scheme 1). To the best of our knowledge, the use of starch sulfuric acid (SSA) as a catalyst for the synthesis of dihydropyrimidinones previously has not been reported.

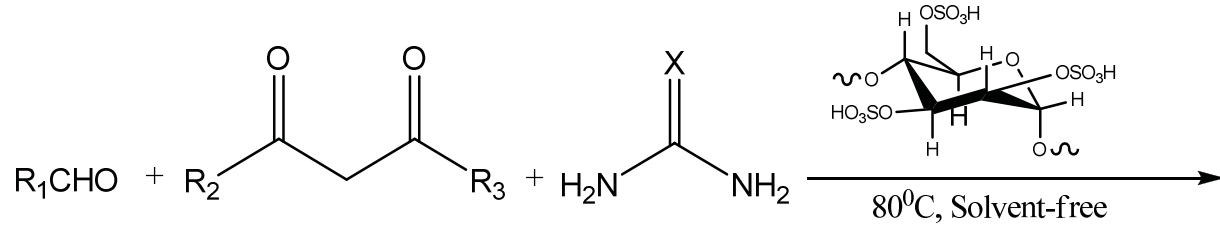

$\mathrm{X}=\mathrm{O}, \mathrm{S}$<smiles>[R]C(=O)C1=C([R])NC([X])NC1[R]</smiles>

Scheme 1. 


\section{Experimental}

All chemicals and analytical grade solvents were purchased from Merck or Fluka chemical company. Melting points were determined in open glass capillaries on Mettler 9100 melting point apparatus. Infrared (IR) spectra were recorded using a 4300 Shimadzu FT-IR spectrometer. ${ }^{1} \mathrm{H}$ NMR and ${ }^{13} \mathrm{C}$ NMR spectra were recorded on a Bruker $250 \mathrm{MHz}$ spectrometer. Mass spectra were recorded on a Shimadzu QP 1100 BX mass spectrometer. All products were known compounds and identified by comparing their physical data to their authentic samples.

\section{General procedure for the Preparation of starch sulfuric acid}

To a magnetically stirred mixture of $5.0 \mathrm{~g}$ of starch in $20 \mathrm{~mL}$ of n-hexane, $1.0 \mathrm{~g}$ of chlorosulfonic acid $(9 \mathrm{mmol})$ was added drop wise at $0{ }^{\circ} \mathrm{C}$ during $2 \mathrm{~h}$. $\mathrm{HCl}$ gas was removed from the reaction vessel immediately. After the addition was complete, the mixture was stirred for $2 \mathrm{~h}$. Then the mixture was filtered and washed with $30 \mathrm{~mL}$ of acetonitrile and dried at room temperature to afford $5.25 \mathrm{~g}$ of starch sulfuric acid as a white powder. Sulfur content of the samples by conventional elemental analysis, was $0.55 \mathrm{mmol} / \mathrm{g}$ for starch sulfuric acid. The number of $\mathrm{H}^{+}$sites on the starch- $\mathrm{SO}_{3} \mathrm{H}$ was determined by acid-base titration was $0.50 \mathrm{meq} / \mathrm{g}$. This value corresponds to about $90 \%$ of the sulfur content, indicating that most of the sulfur species on the sample are in the form of the sulfonic acid groups.

\section{General procedure for the synthesis of dihydropyrimidinones}

SSA $(0.05 \mathrm{~g})$ was added to a mixture of aldehyde $(1 \mathrm{mmol}), \beta$-dicarbonyl compound $(1 \mathrm{mmol})$ and urea or thiourea $(1.5 \mathrm{mmol})$. The neat reaction mixture was heated with stirring for appropriate time at $80{ }^{\circ} \mathrm{C}$ (progress of the reaction was monitored by TLC). At the end of reaction, SSA washed and filtered off with water and ethanol to remove urea (or thiourea) from the surface of the catalyst. Then, the catalyst dried and was maintained for new runs. The filtrate was concentrated and the crude product was recrystallized from ethanol to afford the pure product. Products were identified by comparison with melting points of the authentic compounds. The isolated catalyst was dried at $70{ }^{\circ} \mathrm{C}$ overnight and was reused in the next runs without further purification. The catalyst could be reused at least three times without an appreciable decrease of the yield and reaction rate.

\section{Results and discussion}

SSA is readily prepared by the dropwise addition of chlorosulfonic acid to mixture of starch in n-hexane at $0{ }^{\circ} \mathrm{C}$. It is important to note that, this reaction is easy and clean without any work-up procedure due to $\mathrm{HCl}$ gas is evolved from the reaction vessel immediately. This white homogeneous, nonhygroscopic solid acid is stable under reaction conditions (Scheme 2).

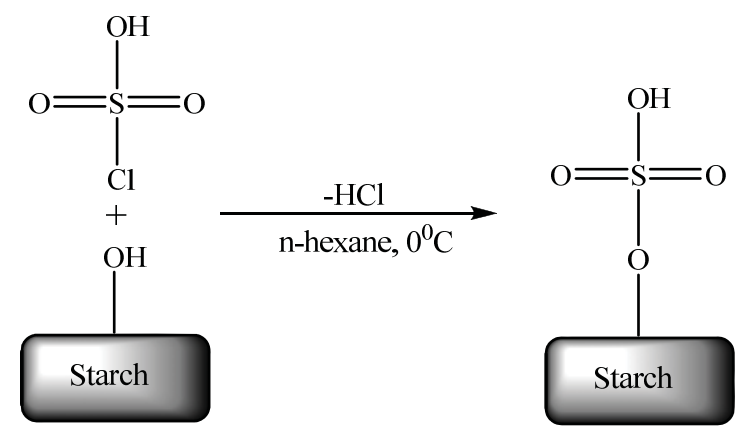

Scheme 2.

We are interested in studying Biginelli reaction with the aim to develop an operationally simple method for the synthesis of some DHPMs. We started our study of the one-pot three-component Biginelli condensation using SSA as the catalyst (Scheme 1), by examining the conditions for the reaction using benzaldehyde, ethylacetoacetate and urea to afford the corresponding DHPM product. In order to optimize the reaction conditions we conducted this reaction in different solvents (Table 1). By using water and ethanol as solvent, the catalyst led to the good conversion but in longer reaction times. The best results were obtained under solvent-free conditions yielding Biginelli products in excellent conversion in shorter reaction times (entry 7, Table 1). No product was formed in the absence of the catalyst (entry 5 , Table 1) whereas in the presence of starch sulfuric acid $(0.05 \mathrm{~g})$, under the same conditions yield increased to $60 \%$ (entry 6, Table 1). The yield of dihydropyrimidinone increased with increasing the amounts of the catalyst from 0.05 to $0.1 \mathrm{~g}$. The use of $0.15 \mathrm{~g}$ of SSA permitted the reaction time to be decreased to $5 \mathrm{~min}$, the yield unexpectedly decreased to $75 \%$ (entry 8, Table 1). A possible explanation for the low product yield is that the starting material or the product may have been destroyed during the reaction when an excess amount $(0.15 \mathrm{~g})$ of SSA was used in the exothermic reaction and 
that $0.1 \mathrm{~g}$ SSA was sufficient to catalyze the reaction effectively. The reaction temperature was also optimized, below $80{ }^{\circ} \mathrm{C}$ the reaction proceeded slow giving a relatively low yield and no improvement was observed above $80{ }^{\circ} \mathrm{C}(\mathrm{entries}$ 9-10, Table 1). All further studies were carried out under solvent-free conditions with $0.1 \mathrm{~g}$ catalyst at $80^{\circ} \mathrm{C}$. Varying the amount of reactants, the best result was obtained when the molar ratio of benzaldehyde, ethyl acetoacetate and urea was $1.0: 1.0: 1.2$.

Effect of solvents on Biginelli reaction of benzaldehyde (1 mmol), ethyl acetoacetate (1 mmol),

Table 1. urea (1.2 mmol), over starch sulfuric acid.

\begin{tabular}{|c|c|c|c|c|c|}
\hline Entry & Solvent & $\mathrm{T}\left({ }^{\circ} \mathrm{C}\right)$ & Catalyst $(\mathrm{g})$ & Time $(\mathrm{min})$ & Yield $(\%)$ \\
\hline 1 & Acetonitrile & Reflux & 0.1 & 150 & 80 \\
\hline 2 & Ethanol & Reflux & 0.1 & 45 & 85 \\
\hline 3 & THF & Reflux & 0.1 & 120 & 50 \\
\hline 4 & Water & Reflux & 0.1 & 30 & 85 \\
\hline 5 & Solvent-free & 80 & - & 120 & 0 \\
\hline 6 & Solvent-free & 80 & 0.05 & 120 & 90 \\
\hline 7 & Solvent-free & 80 & 0.1 & 8 & 75 \\
\hline 8 & Solvent-free & 80 & 0.15 & 5 & 35 \\
\hline 9 & Solvent-free & r.t & 0.1 & 180 & 50 \\
\hline 10 & Solvent-free & 60 & 0.1 & 30 & 50 \\
\hline
\end{tabular}

The structural variations in the aldehydes had no significant effect on the yield and with aldehydes bearing sensitive functional groups like $\mathrm{Cl}, \mathrm{NO}_{2}$, and $\mathrm{OCH}_{3}$ the reaction proceeded smoothly to afford the corresponding products in excellent yields (entries2-4 Table 2). SSA also worked well even with an acid-sensitive aldehyde such as furfural without leading to the formation of any side products (entry 7, Table 2). The reaction of other 1,3-dicarbonyl compounds such as acetylacetone was also run with benzaldehyde and urea in the presence of SSA under solvent- free conditions and the corresponding dihydropyrimidinone was obtained in high yields (entry 8 Table 2). Thiourea has been also used with success to provide the corresponding dihydropyrimidin-2-(1H)-thiones in high yields (entries 9-11, Table 2). It is well known that for Biginelli reaction, aromatic aldehyde works very well, but aliphatic aldehyde works hard, however it is noteworthy that catalyzed the reaction with aliphatic aldehyde. Reaction was very slow and practically did not give any product at $80^{\circ} \mathrm{C}$.

Synthesis of 3,4-dihydropyrimidin-2 (1H)-ones, catalyzed by starch sulfuric acid.

\begin{tabular}{|c|c|c|c|c|c|c|c|c|}
\hline \multirow{2}{*}{ Entry } & \multirow{2}{*}{$\mathbf{R}_{\mathbf{1}}$} & \multirow{2}{*}{$\mathbf{R}_{2}$} & \multirow{2}{*}{$\mathbf{R}_{3}$} & $\mathbf{X}$ & \multirow{2}{*}{$\begin{array}{c}\text { Yield } \\
\mathbf{( \% )}\end{array}$} & $\begin{array}{c}\text { Time } \\
(\mathbf{m i n})\end{array}$ & \multicolumn{2}{|c|}{ M.p. $\left({ }^{\circ} \mathbf{C}\right)$} \\
\hline 1 & $\mathrm{C}_{6} \mathrm{H}_{5}$ & $\mathrm{CH}_{3}$ & $\mathrm{OC}_{2} \mathrm{H}_{5}$ & $\mathrm{O}$ & 85 & 43 & $192-194$ & $198-200[15]$ \\
\hline 2 & $4-\mathrm{ClC}_{6} \mathrm{H}_{4}$ & $\mathrm{CH}_{3}$ & $\mathrm{OC}_{2} \mathrm{H}_{5}$ & $\mathrm{O}$ & 82 & 35 & $208-210$ & $211-213[15]$ \\
\hline 3 & $4-\mathrm{NO}_{2} \mathrm{C}_{6} \mathrm{H}_{4}$ & $\mathrm{CH}_{3}$ & $\mathrm{OC}_{2} \mathrm{H}_{5}$ & $\mathrm{O}$ & 90 & 42 & $200-202$ & $205-207[32]$ \\
\hline 4 & $4-\mathrm{CH}_{3} \mathrm{OC}_{6} \mathrm{H}_{4}$ & $\mathrm{CH}_{3}$ & $\mathrm{OC}_{2} \mathrm{H}_{5}$ & $\mathrm{O}$ & 80 & 55 & $194-196$ & $201-203[18]$ \\
\hline 5 & $4-\mathrm{CH}_{3} \mathrm{C}_{6} \mathrm{H}_{4}$ & $\mathrm{CH}_{3}$ & $\mathrm{OC}_{2} \mathrm{H}_{5}$ & $\mathrm{O}$ & 87 & 52 & $210-212$ & $212-214[25]$ \\
\hline 6 & $\mathrm{Ph} \mathrm{CH}_{\mathrm{C}} \mathrm{CH}$ & $\mathrm{CH}_{3}$ & $\mathrm{OC}_{2} \mathrm{H}_{5}$ & $\mathrm{O}$ & 77 & 62 & $226-228$ & $232-235[19]$ \\
\hline 7 & $2-\mathrm{Furyl}$ & $\mathrm{CH}_{3}$ & $\mathrm{OC}_{2} \mathrm{H}_{5}$ & $\mathrm{O}$ & 90 & 70 & $195-197$ & $201-203[36]$ \\
\hline 8 & $\mathrm{C}_{6} \mathrm{H}_{5}$ & $\mathrm{CH}_{3}$ & $\mathrm{CH}_{3}$ & $\mathrm{O}$ & 86 & 46 & $228-230$ & $233-236[37]$ \\
\hline 9 & $\mathrm{C}_{6} \mathrm{H}_{5}$ & $\mathrm{CH}_{3}$ & $\mathrm{OC}_{2} \mathrm{H}_{5}$ & $\mathrm{~S}$ & 84 & 48 & $204-206$ & $208-210[5]$ \\
\hline 10 & $4-\mathrm{ClC}_{6} \mathrm{H}_{4}$ & $\mathrm{CH}_{3}$ & $\mathrm{OC}_{2} \mathrm{H}_{5}$ & $\mathrm{~S}$ & 92 & 40 & $214-216$ & $208-210[38]$ \\
\hline 11 & $4-\mathrm{CH}_{3} \mathrm{OC}_{6} \mathrm{H}_{4}$ & $\mathrm{CH}_{3}$ & $\mathrm{OC}_{2} \mathrm{H}_{5}$ & $\mathrm{~S}$ & 80 & 49 & $143-145$ & $150-152[5]$ \\
\hline
\end{tabular}

The mechanism of the Biginelli reaction established by Kappe proposed that the key step in this cyclocondensation process should involve the formation of $\mathrm{N}$-acyliminium ion intermediate. The suggested mechanism for the Biginelli reaction catalyzed by SSA under solvent-free conditions is outlined in Scheme 3. 
A comparative study was performed for the use of SSA with some of the reported catalysts for the Biginelli reaction (Table 3). In most methods, the reaction was performed in solvent such as acetic acid, dioxane and toluene. Thus, SSA promoted the reactions more effectively than the other catalysts and should be considered as one of the best choice for selecting an economically convenient, user friendly catalyst.

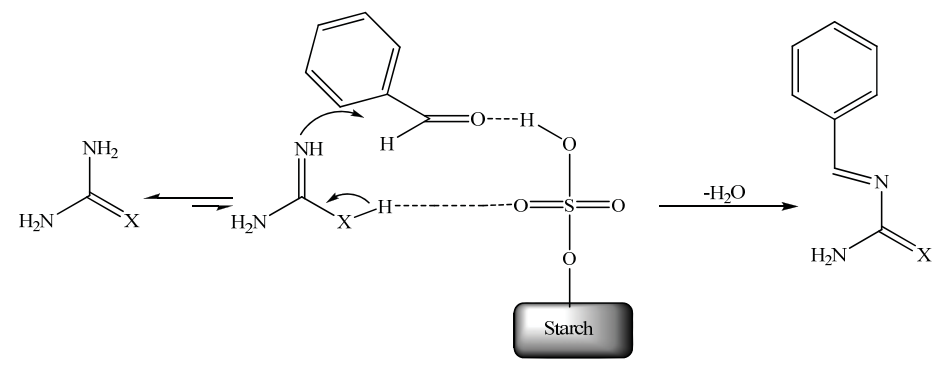

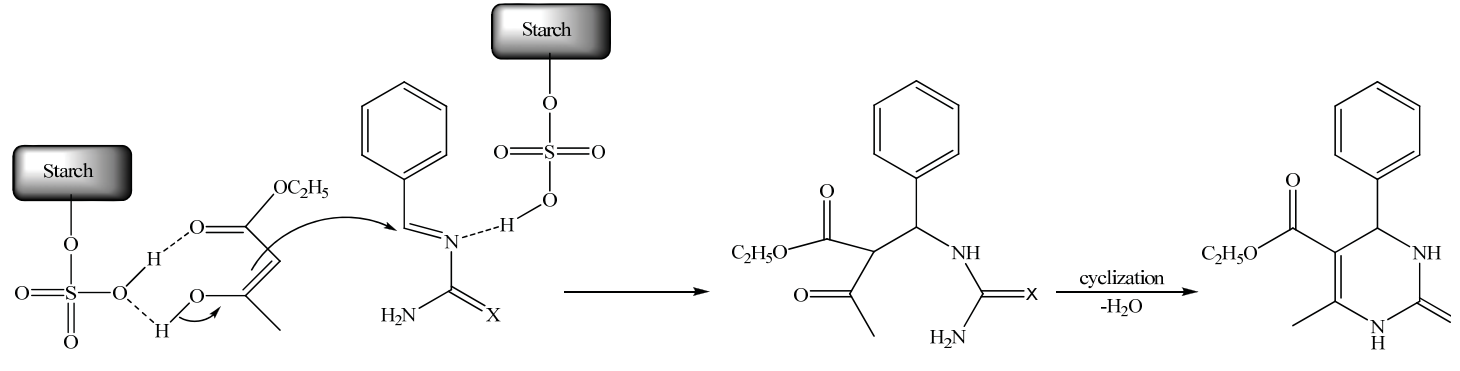

Scheme 3.

Biginelli reaction with benzaldehyde, ethylacetoacetate and urea with different catalysts.

\begin{tabular}{|c|c|c|c|c|c|}
\hline Entry & Catalyst & Condition & Time & Yield $(\%)$ & References \\
\hline 1 & $\mathrm{TaBr}_{5}$ & Solvent free $/ 100^{\circ} \mathrm{C}$ & $40 \mathrm{~min}$ & 97 & [12] \\
\hline 2 & Trichloroisocynuric acid (TCCA) & Ethanol/ Reflux & $12 \mathrm{~h}$ & 94 & [13] \\
\hline 3 & Montmorillonite KSF & Solvent free $/ 100{ }^{\circ} \mathrm{C}$ & $48 \mathrm{~h}$ & 77 & [17] \\
\hline 4 & $\mathrm{BF}_{3} \cdot \mathrm{OEt}_{2} / \mathrm{Cu}(\mathrm{OAc})_{2}$ & Acetic acid $/ 65^{\circ} \mathrm{C}$ & $18 \mathrm{~h}$ & 71 & {$[18]$} \\
\hline 5 & Silica-sulfuric acid & Ethanol/Reflux/ & $6 \mathrm{~h}$ & 91 & {$[19]$} \\
\hline 6 & $\mathrm{H}_{3} \mathrm{PMo}_{12} \mathrm{O}_{40}$ & Acetic acid/Reflux & $5 \mathrm{~h}$ & 90 & [25] \\
\hline 7 & Bentonite/PS-SO $\mathrm{H}_{3} \mathrm{Hanocomposite}$ & Solvent-free $/ 100{ }^{\circ} \mathrm{C}$ & $30 \mathrm{~min}$ & 89 & {$[28]$} \\
\hline 8 & Sulfated tungstate & Solvent-free $/ 80^{\circ} \mathrm{C}$ & $60 \mathrm{~min}$ & 92 & [29] \\
\hline 9 & Imidazol-1-yl-acetic acid. & Solvent-free $/ 100{ }^{\circ} \mathrm{C}$ & $35 \mathrm{~min}$ & 90 & {$[30]$} \\
\hline 10 & Starch sulfuric acid(SSA) & Solvent free $/ 100^{\circ} \mathrm{C}$ & $10 \mathrm{~min}$ & 90 & This work \\
\hline
\end{tabular}

In conclusion, we have shown an efficient SSA catalyzed one-pot synthesis of 3,4-dihydropyrimidin-2- (1H)-ones and thione analogs by multicomponent Biginelli reactions under solvent-free conditions, using commercially available substrates. The attractive features of this protocol are its green-ness with respect to solvent-free reaction, recyclability of catalyst, mild reaction conditions, short reaction times and high yield.

\section{The spectral data}

5-(Ethoxycarbonyl)-6-methyl-4-phenyl-3,4-dihydropyrimidin-2(1H)-one (Table 2, entry 1): IR (KBr): 3240, 1725, 1635 $\mathrm{cm}^{-1} .{ }^{1} \mathrm{H}$ NMR (DMSO-d $): \delta=9.20(\mathrm{~s}, 1 \mathrm{H}, \mathrm{NH}), 7.75(\mathrm{~s}, 1 \mathrm{H}, \mathrm{NH}), 7.10-7.28(\mathrm{~m}, 5 \mathrm{H}, \operatorname{arom~CH}), 5.14(\mathrm{~s}, 1 \mathrm{H}, \mathrm{CH}), 3.97$ $\left(\mathrm{q}, J=7.5 \mathrm{~Hz}, 2 \mathrm{H}, \mathrm{OCH}_{2}\right), 2.25\left(\mathrm{~s}, 3 \mathrm{H}, \mathrm{CH}_{3}\right), 1.09$ (t, $\left.J=7.5 \mathrm{~Hz}, 3 \mathrm{H}, \mathrm{OCH}_{2} \mathrm{CH}_{3}\right) .{ }^{13} \mathrm{C}-\mathrm{NMR}\left(\mathrm{DMSO}-\mathrm{d}_{6}\right) \delta: 14.11,17.94$,

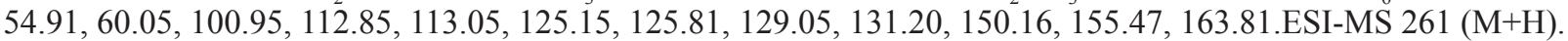

5-(Ethoxycarbonyl)-6-methyl-4-(4-chlorophenyl)-3,4-dihydropyrimidin-2(1H)-one (Table 2, entry 2): IR (KBr): 3240, 1723, $1643 \mathrm{~cm}^{-1} .{ }^{1} \mathrm{H}$ NMR (DMSO-d 6$): \delta=9.26(\mathrm{~s}, 1, \mathrm{NH}), 7.79(\mathrm{~s}, 1 \mathrm{H}, \mathrm{NH}), 7.40-7.30$ (m, 4H, arom CH), $5.14(\mathrm{~s}, 1 \mathrm{H}$, 
CH), 3.99 (q, $J=7.5 \mathrm{~Hz}, 2 \mathrm{H}, \mathrm{OCH}_{2}$ ), $2.25\left(\mathrm{~s}, 3 \mathrm{H}, \mathrm{CH}_{3}\right), 1.09$ (t, $J=7.5 \mathrm{~Hz}, 3 \mathrm{H}, \mathrm{OCH}_{2} \mathrm{CH}_{3}$ ). ${ }^{13} \mathrm{C}-\mathrm{NMR}\left(\mathrm{DMSO}-\mathrm{d}_{6}\right) \delta$ : $14.18,18.62,55.72,60.21,101.55,118.17,130.32,142.29,152.31,153.39,159.17,165.83$.ESI-MS $295(\mathrm{M}+\mathrm{H})$.

5-(Ethoxycarbonyl)-6-methyl-4-(4-nitrophenyl)-3,4-dihydropyrimidin-2(1H)-one (Table 2, entry 3): IR (KBr): 3239, 1724, 1645, $\mathrm{cm}^{-1} .{ }^{1} \mathrm{H}$ NMR (DMSO-d $)$ ): $\delta=9.27(\mathrm{~s}, 1 \mathrm{H}, \mathrm{NH}), 8.20(\mathrm{~d}, J=9.15 \mathrm{~Hz}, 2 \mathrm{H}$, arom CH), $7.91(\mathrm{~s}, 1 \mathrm{H}, \mathrm{NH})$, $7.50\left(\mathrm{~d}, J=9.18 \mathrm{~Hz}, 2 \mathrm{H}\right.$, arom CH), $5.18(\mathrm{~d}, J=2.25 \mathrm{~Hz}, 1 \mathrm{H}, \mathrm{CH}), 3.79\left(\mathrm{q}, J=7.50 \mathrm{~Hz}, 2 \mathrm{H}, \mathrm{OCH}_{2}\right), 2.25(\mathrm{~s}, 3 \mathrm{H}$, $\left.\mathrm{OCH}_{2} \mathrm{CH}_{3}\right), 1.10\left(\mathrm{t}, J=7.50 \mathrm{~Hz}, 3 \mathrm{H}, \mathrm{CH}_{3}\right) \cdot{ }^{13} \mathrm{C}-\mathrm{NMR}\left(\mathrm{DMSO}_{\mathrm{d}}\right) \mathrm{d}$ ) $\delta: 14.22,18.71,55.81,60.15,101.60,118.15,130.37$, 138.34, 152.26, 153.41, 159.15, 165.85.ESI-MS $306(\mathrm{M}+\mathrm{H})$.

5-(Ethoxycarbonyl)-4-(4-methoxyphenyl)-6-methyl-3,4-dihydropyrimidine-2(1H)-one (Table 2, entry 4): IR (KBr): $3241,1718,1636 \mathrm{~cm}^{-1} .{ }^{1} \mathrm{H}$ NMR (DMSO-d $\left.{ }_{6}\right): \delta=9.15(\mathrm{~s}, 1 \mathrm{H}, \mathrm{NH}), 7.76(\mathrm{~s}, 1 \mathrm{H}, \mathrm{NH}), 7.15(\mathrm{~d}, J=8.65 \mathrm{~Hz}, 2 \mathrm{H}$, arom CH), $6.92\left(\mathrm{~d}, J=8.65 \mathrm{~Hz}, 2 \mathrm{H}\right.$, arom CH), $5.12(\mathrm{~s}, 1 \mathrm{H}, \mathrm{CH}), 3.96\left(\mathrm{q}, J=7.50 \mathrm{~Hz}, 2 \mathrm{H}, \mathrm{OCH}_{2}\right), 3.76\left(\mathrm{~s}, 3 \mathrm{H}, \mathrm{C}_{6} \mathrm{H}_{4}-\mathrm{OCH}_{3}\right)$, $2.24\left(\mathrm{~s}, 3 \mathrm{H}, \mathrm{CH}_{3}\right), 1.10\left(\mathrm{t}, J=7.50 \mathrm{~Hz}, 3 \mathrm{H}, \mathrm{CH}_{3}\right) .{ }^{13} \mathrm{C}-\mathrm{NMR}$ (DMSO-d6) $\delta: 14.32,18.80,55.23,55.40,60.17,101.68$, $114.06,127.97,136.22,146.16,153.59,159.30,165.87$. ESI-MS $291(\mathrm{M}+\mathrm{H})$.

5-(Ethoxycarbonyl)-6-methyl-4-(4-methylphenyl)-3,4-dihydropyrimidin-2(1H)-one (Table 2, entry 5): IR (KBr): 3242, $1715,1633 \mathrm{~cm}^{-1} .{ }^{1} \mathrm{H}$ NMR (DMSO-d $): \delta=9.16(\mathrm{~s}, 1 \mathrm{H}, \mathrm{NH}), 7.80(\mathrm{~s}, 1 \mathrm{H}, \mathrm{NH}), 7.16-7.12(\mathrm{~m}, 4 \mathrm{H}$, arom CH), 5.09 $(\mathrm{s}, 1 \mathrm{H}, \mathrm{CH}), 3.96$ (q, $\left.J=7.50 \mathrm{~Hz}, 2 \mathrm{H}, \mathrm{OCH}_{2} \mathrm{CH}_{3}\right), 2.27\left(\mathrm{~s}, 3 \mathrm{H}, \mathrm{C}_{6} \mathrm{H}_{4}-\mathrm{CH}_{3}\right), 2.21\left(\mathrm{~s}, 3 \mathrm{H}, \mathrm{CH}_{3}\right), 1.08$ (t, $J=7.50 \mathrm{~Hz}$, $3 \mathrm{H}, \mathrm{OCH}_{2} \mathrm{CH}_{3}$ ). ${ }^{13} \mathrm{C}-\mathrm{NMR}\left(\mathrm{DMSO}-\mathrm{d}_{6}\right) \delta: 14.25,17.62,53.20,61.75,106.48,115.16,126.87,136.52,147.38,150.25$, 158.35, 167.80.ESI-MS $275(\mathrm{M}+\mathrm{H})$.

5-(Ethoxycarbonyl)-6-methyl-4-styryl-3,4-dihydropyrimidin-2(1H)-one (Table 2, entry 6): IR (KBr): 3246, 1704, 1650 $\mathrm{cm}^{-1} .{ }^{1} \mathrm{H}$ NMR (DMSO-d $)$ ): $\delta=9.12(\mathrm{~s}, 1 \mathrm{H}, \mathrm{NH}), 7.79(\mathrm{~s}, 1 \mathrm{H}, \mathrm{NH}), 7.42-7.25(\mathrm{~m}, 5 \mathrm{H}, \operatorname{arom~CH}), 6.33(\mathrm{~d}, J=15.90 \mathrm{~Hz}$, $1 \mathrm{H}, \mathrm{HC}=\mathrm{CH}), 6.20(\mathrm{~d}, J=15.80 \mathrm{~Hz}, 1 \mathrm{H}, \mathrm{CH}=\mathrm{CH}), 4.74(\mathrm{~d}, J=4.80 \mathrm{~Hz}, 1 \mathrm{H}, \mathrm{CH}), 4.09\left(\mathrm{q}, J=7.50 \mathrm{~Hz}, 2 \mathrm{H}, \mathrm{OCH}_{2}\right), 2.20$ (s, $3 \mathrm{H}, \mathrm{CH}_{3}$ ), 1.10 (t, $J=7.50 \mathrm{~Hz}, 3 \mathrm{H}, \mathrm{OCH}_{2} \mathrm{CH}_{3}$ ). ${ }^{13} \mathrm{C}-\mathrm{NMR}\left(\mathrm{DMSOd}_{6}\right) \delta: 14.21,17.31,51.84,59.45,98.54,127.34$, $128.54,129.54,130.59,131.24,135.24,145.34,153.62,165.23$.ESI-MS $287(\mathrm{M}+\mathrm{H})$.

5-(Ethoxycarbonyl)-4-(2-furyl)-6-methyl-3,4-dihydropyrimidin-2(1H)-one (Table 2, entry 7): IR (KBr): 3239, 1705, $1644 \mathrm{~cm}^{-1}$. ${ }^{1} \mathrm{H}$ NMR: $\delta=9.22(\mathrm{~s}, 1 \mathrm{H}, \mathrm{NH}), 7.74(\mathrm{~s}, 1 \mathrm{H}, \mathrm{NH}), 7.53(\mathrm{~d}, J=6.0 \mathrm{~Hz}, 1 \mathrm{H}$, furyl CH), 6.30-6.08 (d, $J=3.0$ $\mathrm{Hz}, 2 \mathrm{H}$, furyl-CH), $5.20(\mathrm{~s}, 1 \mathrm{H}, \mathrm{CH}), 4.02\left(\mathrm{q}, J=7.50 \mathrm{~Hz}, 2 \mathrm{H}, \mathrm{CH}_{2} \mathrm{CH}_{3}\right), 2.22\left(\mathrm{~s}, 3 \mathrm{H}, \mathrm{CH}_{3}\right), 1.12(\mathrm{t}, J=7.50 \mathrm{~Hz}, 3 \mathrm{H}$, $\left.\mathrm{OCH}_{2} \mathrm{CH}_{3}\right) .{ }^{13} \mathrm{C}-\mathrm{NMR}\left(\mathrm{DMSOd}_{6}\right) \delta: 14.15,17.55,54.33,61.44,106.45,106.72,110.36,142.25,147.38,150.28,152.55$, 167.62.ESI-MS $251(\mathrm{M}+\mathrm{H})$.

5-Aceto-6-methyl-4-phenyl-3,4-dihydropyrimidin-2(1H)-one (Table 2, entry 8): IR (KBr): $3241,1715,1643 \mathrm{~cm}^{-1} \cdot{ }^{1} \mathrm{H}$ NMR: $\delta=9.20(\mathrm{~s}, 1 \mathrm{H}, \mathrm{NH}), 7.76(\mathrm{~s}, 1 \mathrm{H}, \mathrm{NH}), 7.35-7.25(\mathrm{~m}, 5 \mathrm{H}$, arom $\mathrm{CH}), 5.25(\mathrm{~s}, 1 \mathrm{H}, \mathrm{CH}), 2.24\left(\mathrm{~s}, 3 \mathrm{H}, \mathrm{CH}_{3} \mathrm{CO}\right)$, 2.07 (s, $3 \mathrm{H}, \mathrm{CH}_{3}$ ). ${ }^{13} \mathrm{C}-\mathrm{NMR}\left(\mathrm{DMSOd}_{6}\right) \delta: 18.65,52.32,54.70,108.47,113.36,122.41,131.17,130.51,154.11,160.20$, 164.42.ESI-MS $247(\mathrm{M}+\mathrm{H})$.

5-(Ethoxycarbonyl)-6-methyl-4-phenyl-3,4-dihydropyrimidin-2(1H)-thione (Table 2, entry 9): IR (KBr): 3258, 1671, $1576 \mathrm{~cm}^{-1}$. ' $\mathrm{H}$ NMR: $\delta=10.33(\mathrm{~s}, 1 \mathrm{H}, \mathrm{NH}), 9.64(\mathrm{~s}, 1 \mathrm{H}, \mathrm{NH}), 7.35-7.19\left(\mathrm{~m}, 5 \mathrm{H}, \mathrm{C}_{6} \mathrm{H}_{5}\right), 5.16(\mathrm{~d}, \mathrm{~J}=3.50 \mathrm{~Hz}, 1 \mathrm{H}, \mathrm{CH})$, $4.05\left(\mathrm{q}, \mathrm{J}=7.20 \mathrm{~Hz}, 2 \mathrm{H}, \mathrm{OCH}_{2} \mathrm{CH}_{3}\right), 2.28\left(\mathrm{~s}, 3 \mathrm{H}, \mathrm{CH}_{3}\right), 1.09\left(\mathrm{t}, \mathrm{J}=7.20 \mathrm{~Hz}, 3 \mathrm{H}, \mathrm{OCH}_{2} \mathrm{CH}_{3}\right){ }^{13} \mathrm{C}-\mathrm{NMR}\left(\mathrm{DMSOd}_{6}\right) \delta$ : $14.23,17.91,54.85,60.15,100.90,112.84,115.12,125.15,126.85,129.64,131.45,150.27,162.63,180.25$.ESI-MS $277(\mathrm{M}+\mathrm{H})$.

4-(4-Chlorophenyl)-5-ethoxycarbonyl-6-methyl-3,4-dihydropyrimidin-2(1H)-thione (Table 2, entry 10):

IR (KBr): 3255, 1657, $1560 \mathrm{~cm}^{-1} .{ }^{1} \mathrm{H}$ NMR: $\delta=10.36$ (s, $\left.1 \mathrm{H}, \mathrm{NH}\right), 9.65$ (s, $\left.1 \mathrm{H}, \mathrm{NH}\right), 7.43-7.19\left(\mathrm{~m}, 4 \mathrm{H}, \mathrm{C}_{6} \mathrm{H}_{4}\right)$,

$5.16(\mathrm{~s}, 1 \mathrm{H}, \mathrm{CH}), 4.00\left(\mathrm{q}, \mathrm{J}=6.50 \mathrm{~Hz}, 2 \mathrm{H}, \mathrm{OCH}_{2} \mathrm{CH}_{3}\right), 2.25\left(\mathrm{~s}, 3 \mathrm{H}, \mathrm{CH}_{3}\right), 1.08\left(\mathrm{t}, \mathrm{J}=6.50 \mathrm{~Hz}, 3 \mathrm{H}, \mathrm{OCH}_{2} \mathrm{CH}_{3}\right) .{ }^{13} \mathrm{C}-\mathrm{NMR}$ $\left(\operatorname{DMSOd}_{6}\right)$ ): 14.22, 18.35, 58.37, 61.73, 104.25, 126.42, 128.44, 132.72, 141.48, 160.37, 167.25, 174.18.ESI-MS 311 $(\mathrm{M}+\mathrm{H})$.

5-Ethoxycarbonyl-4-(4- methoxyphenyl)-6-methyl-3,4-dihydropyrimidin-2(1H)-thione (Table 2, entry 11):

IR (KBr): 3250, 1651, 1598, $1561 \mathrm{~cm}^{-1} .{ }^{1} \mathrm{H}$ NMR: $\delta=10.29(\mathrm{~s}, 1 \mathrm{H}, \mathrm{NH}), 9.59(\mathrm{~s}, 1 \mathrm{H}, \mathrm{NH}), 7.14-6.87\left(\mathrm{~m}, 4 \mathrm{H}, \mathrm{C}_{6} \mathrm{H}_{4}\right)$, $5.10(\mathrm{~s}, 1 \mathrm{H}, \mathrm{CH}), 3.95\left(\mathrm{q}, \mathrm{J}=7.25 \mathrm{~Hz}, 2 \mathrm{H}, \mathrm{OCH}_{2} \mathrm{CH}_{3}\right), 3.71\left(\mathrm{~s}, 3 \mathrm{H}, \mathrm{OCH}_{3}\right), 2.27\left(\mathrm{~s}, 3 \mathrm{H}, \mathrm{CH}_{3}\right), 1.09$ (t, J=7.25Hz, $3 \mathrm{H}$, $\left.\mathrm{OCH}_{2} \mathrm{CH}_{3}\right) .{ }^{13} \mathrm{C}-\mathrm{NMR}\left(\mathrm{DMSOd}_{6}\right) \delta: 14.32,18.05,55.24,55.49,60.45,101.84,114.32,127.74,137.25,147.15,159.45$, 165.62, 182.48.ESI-MS $307(\mathrm{M}+\mathrm{H})$.

\section{References}

[1] Kappe, C.O. Acc, Chem. Res. 2000, 33, 879.

[2] Kappe, C.O. Tetrahedron.1993, 49, 6937.

[3] Kappe, C.O. Eur. J. Med. Chem. 2000, 35, 1043.

[4] Biginelli, P. Gazz. Chim. Ital. 1983, 23, 360.

[5] Khabazzadeh, H.; Saidi, K.; Sheibani, H. Bioorg. Med, Chem. Lett. 2008, 18, 278.

[6] Jain, S.L.; Prasad, V.V.D.N.; Sain, B. Catal. Commun.2008, 9, 499.

[7] Cepanec, I.; Litvić, M.; Filipan- Litvić, M.; Grüngold, I. Tetrahedron. 2007, 63, 11822.

[8] Kamal, A.; Krishnaji, T.; Azhar, M.A. Catal. Commun, 2007, 8, 1929.

[9] Banik, B.K.; Reddy, A.T.; Datta, A.; Mukhopadhyay, C. Tetrahedron Lett, 2007, 48, 7392. 
[10] Adibi, H.; Samimi, H.A.; Beygzadeh, M. Catal. Commun.2007, 8, 2119.

[11] Nandurkar, N.S.; Bhanushali, M.J.; Bhor, M.D.; Bhanage, B.M. J. Mol. Catal. A: Chem. 2007, $14,271$.

[12] Ahmed, N.; Lier, J.E. Tetrahedron Lett. 2007, 48, 5407.

[13] Bigdeli, M.A.; Jafari, S.; Mahdavinia, G.H.; Hazarkhani, H. Catal. Commun. 2007, 8, 1641.

[14] Polshettiwar, V.; Varma, R.S. Tetrahedron Lett. 2007, 48, 7343.

[15] Yu, Y.; Liu, D.; Liu, C.; Luo, G. Bioorg. Med. Chem. Lett. 2007, 17, 3508.

[16] Jin, T.; Zhang, S.; Li, T. Synth. Commun. 2002, 32, 1847.

[17] Bigi, F.; Carloni, S.; Frullanti, B.; Maggi, R.; Sartori, G. Tetrahedron Lett. 1999, 40, 3465.

[18] Hu, E.H.; Sidler, D.R.; Dolling, U.-H. J. Org. Chem. 1998, 63, 3453.

[19] Salehi, P.; Dabiri, M.; Zolfigol, M.A.; Bodaghi Fard, M.A. Tetrahedron Lett. 2003, 44, 2889.

[20] Hassani, Z.; Islami, M.R.; Kalantari, M. Bioorg. Med. Chem. Lett. 2006, 16, 4479.

[21] Ismaili, L.; Nadaradjane, A.; Nicod, L.; Guyon, C.; Xicluna, A.; Robert, J.F.; Refouvelet, B. Eur. J. Med. Chem. 2007, 1 .

[22] Chen, W.-Y.; Qin, S.; Jin, J.R. Catal. Commun. 2007, 8, 123.

[23] Chen, X.-H.; Xu, X.-Y.; Liu, H.; Cun, L.-F.; Gong, L.-Z. J. Am. Chem. Soc. 2006, 128, 14802.

[24] Heravi, M.M.; Derikvand, F.; Bamoharram, F.F. J. Mol. Cata. A: Chem. 2005, 242, 173.

[25] Heravi, M.M.; Bakhtiari, K.; Bamoharram, F.F. Catal. Commun.2006, 7, 373.

[26] Sharghi, H.; Jokar, M. Synth. Commun. 2009, 50, 958.

[27] Narahari, S.R.; Reguri, B.R.; Gudaparthi, O.; Mukkanti, K. Tetrahedron Lett. 2012, 53, 1543.

[28] Kalbasi, R.J.; Massah, A.R.; Daneshvarnejad, B. Appl. Clay. Sci. 2012, 55, 1.

[29] Salim, S.D.; Akamanchi, K.G. Cataly. Commun. 2011, 12, 1153.

[30] Kargar, M.; Hekmatshoar, R.; Mostashari, A.; Hashemi, Z. Cataly. Commun. 2011, 15, 123.

[31] Moosavifar, M. C. R. Chimie. 2012, 15, 444.

[32] Shaabani, A.; Bazgir, A.; Teimuri, F. Tetrahedron Lett. 2003, 44, 857.

[33] Shirini, F.; Marjani, K.; Taherpour Nahzomi, H. Arkivoc. 2007, 51.

[34] Bigi, F.; Carloni, S.; Frullanti, B.; Maggi, R.; Sartori, G. Tetrahedron Lett. 1999, 40, 3465.

[35] Yadav, J.S.; Reddy, B.V.S.; Srinivas, R.; Venogopal, C.; Ramalingam, T. Synthesis. 2001, 1341.

[36] Lee, K.Y.; Ko, L.Y. Bull. Korean Chem. Soc. 2004, 25, 1929.

[37] Fu, N.F. Yuan, Y.F. Cao, Z. Wang, Z.W. Wang, J.T. Peppe, C. Tetrahedron. 2002, 58, 4801.

[38] Xue, S.; Shen, Y.C.; Li, Y.L.; Shen, X.M.; Guo, Q.X. Chin. J. Chem. 2002, 20, 385.

[39] Clark, J.H.; Macquarrie, D.J. Green Chemistry and Technology, Blackwell, Abingdon, 2002.

[40] Tomasik. P. Chemical and Functional Properties of Food Saccharides; CRC Press; 2004.

[41] Morris; D.L. Science. 1948, 107, 254. 